Irish Math. Soc. Bulletin

Number 88, Winter 2021, 47-55

ISSN 0791-5578

\title{
On p-adic modules with isomorphic endomorphism algebras
}

\author{
BRENDAN GOLDSMITH AND NOEL WHITE
}

\begin{abstract}
We investigate pairs of modules over the ring of p-adic integers having isomorphic endomorphism algebras. In many cases this forces the modules to be isomorphic but there are two exceptional situations where isomorphism does not follow.
\end{abstract}

\section{INTRODUCTION}

Throughout this work we shall focus on modules over the ring $R$ of $p$-adic integers, where $p$ is a fixed but arbitrary prime. Recall that elements of $R$ may be considered as infinite sums $\sum_{i=0}^{\infty} r_{i} p^{i}$, where each $r_{i}$ is an integer with $0 \leq r_{i}<p$. Addition and multiplication are defined in the usual way and it is easy to check that $R$ then becomes an integral domain. It is possible to topologise $R$ with a linear topology having the submodules $p^{j} R(j=0,1, \ldots)$ as a base of neighbourhoods of 0 ; the resulting ' $p$ adic topology' is metrisable and $R$ is complete with respect to this topology. $R$ is an example of a complete discrete valuation ring and such rings may be regarded as a not too complicated generalisation of a field. Associated with $R$ we have its field of quotients $Q$ and for each integer $n$, the cyclic module $R / p^{n} R$ which is just the usual additively written cyclic group of integers modulo $p^{n}$. In keeping with the usual notation for Abelian groups, we often write $R\left(p^{n}\right)$ instead of $R / p^{n} R$. There is one further significant module associated with $R$ viz. the quotient module $Q / R$. This module is divisible in the sense that given any element $x$, the equation $x=p^{n} y$ has a solution $y$. It is not difficult to show that $Q / R$ is isomorphic to the usual Prüfer quasi-cyclic group $\mathbb{Z}\left(p^{\infty}\right)$; recall that $\mathbb{Z}\left(p^{\infty}\right)$ is the additively written version of the infinite multiplicative group of all $p^{n}$ th complex roots of unity and is generated by elements $c_{1}, c_{2}, \ldots, c_{n}, \ldots$ such that $p c_{1}=0, p c_{2}=c_{1}, \ldots, p c_{n+1}=c_{n}, \ldots$ Hence each $c_{n}$ has order $p^{n}$ and every element of $\mathbb{Z}\left(p^{\infty}\right)$ is a multiple of some $c_{n}$. Consequently, all proper submodules of $Q / R$ are finite and they form a chain under inclusion. It is well known that every $R$-module $G$ can be expressed as a direct sum $G=D \oplus X$, where $D$ is a divisible module and $X$ is reduced in the sense that $X$ contains no divisible submodules. Furthermore, a divisible $R$-module is of the form $\bigoplus_{\lambda} Q \oplus \bigoplus_{\mu} Q / R$ for cardinals $\lambda, \mu \geq 0$. We shall reserve the notation $R$ for the ring of $p$-adic integers and all modules (unless specified to the contrary) will be left modules over $R$.

It is a long-standing question in algebra as to what extent the algebra of endomorphisms of an Abelian group or module (over an arbitrary ring) determines the module itself. In the simplest case of vector spaces over a field, it is an easy exercise in linear algebra to show that if $V, W$ are finite-dimensional spaces over a field $F$ then $V \cong W$ if and only if the corresponding rings of linear transformations (i.e. the endomorphism rings) are isomorphic as rings. It is less trivial, but nonetheless true, that the corresponding

2020 Mathematics Subject Classification. 20K30, 16A65.

Key words and phrases. p-adic modules, endomorphism algebras.

Received on 1-9-2021; revised 19-11-2021.

DOI:10.33232/BIMS.0088.47.55. 
result remains true if one removes the restriction of finite dimensionality: however, a simplistic appeal to a matrix argument will result in a need for some additional set theory such as the Generalised Continuum Hypothesis (GCH).

The question that we wish to consider in the rest of this paper is simply: If $G, H$ are $R$-modules and the endomorphism algebras $E_{R}(G), E_{R}(H)$ are isomorphic (as $R$ algebras), what can we say about the relationship (if any) between $G$ and $H$ ? Note that in one direction the argument is straightforward (even for modules over an arbitrary ring $S$ ): if $A, B$ are isomorphic $S$-modules via the isomorphism $\phi: A \rightarrow B$, then the mapping $\Phi: E_{S}(A) \rightarrow E_{S}(B)$ defined for an arbitrary endomorphism $\alpha$ of $A$ by $\Phi(\alpha)=\phi \alpha \phi^{-1}$ is easily seen to be an $S$-algebra isomorphism.

It will be clear from our arguments that many of our results can be generalised to include modules over rings other than the rings of $p$-adic integers. However, it is well known that a question such as that above has no hope of being answered in, for example, the category of all $\mathbb{Z}$-modules (Abelian groups). In particular, the situation where torsion-free modules are involved is, in many cases, totally intractable: for example, it is known that there exist arbitrary large torsion-free Abelian groups all having endomorphism ring isomorphic to the ring of integers $\mathbb{Z}$. A specific advantage of working with $p$-adic modules is that the only indecomposable modules are then $Q, R, Q / R$ and $R\left(p^{n}\right)$ for each positive integer $n$. Moreover, the corresponding endomorphism algebras are easily calculated to be precisely $Q, R, R, R\left(p^{n}\right)$; the fact that $E_{R}(R) \cong E_{R}(Q / R)$ will, of course, be a difficulty for us in addressing the relationship between $R$-modules and their endomorphism algebras.

The first significant result in this area is probably that of Baer [1] and this was significantly simplified and generalised by Kaplansky [11, Theorem 28] who showed, inter alia, that torsion $R$-modules have the property that any isomorphism between their endomorphism algebras is induced by an endomorphism of the modules themselves. (Kaplansky's argument worked in the more general context of complete discrete valuation rings.) Consequently, results of this form are often referred to in the literature as 'Baer-Kaplansky Theorems'- see, for example, $[3,12]$ or [14] for a small selection of such results. Subsequently Hauptfleisch [10] and Wolfson [16] extended the result to certain classes of torsion-free Abelian groups and modules; in particular Wolfson showed that if $G, H$ are reduced torsion-free $R$-modules then $E_{R}(G) \cong E_{R}(H)$ if and only if $G \cong H$. The situation for mixed modules and Abelian groups is difficult but some interesting results were obtained in [15] and [4]. Recently the authors, working on an unpublished idea of Corner, have considered the corresponding problem where isomorphism of the endomorphism algebras is replaced by anti-isomorphism, [9].

Our notation is largely standard and this, and relevant ideas from Abelian group and module theory, may be found in the texts [5, 6, 7] or [11]. In particular, for an $R$-module $G, t G$ will denote the torsion submodule of $G: t G=\left\{g \in G \mid p^{n} g=0\right.$, for some $\left.n \geq 0\right\}$.

Mappings are written on the left and, when there is no risk of confusion, we shall denote the $R$-algebra of $R$-endomorphisms of an $R$-module $G$ by simply $E(G)$; in the sequel when we write $E(G) \cong E(H)$ for $R$-modules $G, H$, we shall always mean that the isomorphism is an $R$-algebra isomorphism. In certain special cases we shall also make use of our knowledge of the structure of the module of $R$-homomorphism between modules $G, H$, which we denote $\operatorname{simply}$ by $\operatorname{Hom}(G, H)$ and the main results we need are directly analogous to those that may be found for Abelian groups in [5, Chapter VIII].

In the next section, Preliminaries, we consider the basic ideas centring on the use of idempotents and introduce an ad hoc notion of an endo-root which allows us to present a uniform treatment of some of the simpler cases that we wish to consider; an interesting by-product of this is an easy proof that vector spaces of arbitrary dimension over a fixed 
field are isomorphic if and only if their rings of linear transformations are isomorphic as rings.

In the third section we consider $R$-modules which are not reduced, i.e., modules having a direct summand isomorphic to either $Q$ or $Q / R$. In particular, in this section we are able to show that if $E(H) \cong E(G)$, where $G$ is a divisible $R$-module, then either $H \cong G$ or else $G=\bigoplus_{\mu} Q / R$ and $H=\widehat{\bigoplus_{\mu} R}$, the $p$-adic completion of the free $R$-module of rank $\mu$, an arbitrary cardinal.

In the final section we show that the situation is similar to that for divisible modules but that there is one additional possibility: the modules $G, H$ have isomorphic torsion basic submodules but are not themselves necessarily isomorphic, We indicate by means of an example that the situation is extremely complex which strongly suggests the unlikelihood of obtaining a complete classification of all outcomes.

The material in this work is part of the first chapter of a research thesis being prepared by the second author for submission to TU Dublin.

\section{Preliminaries.}

A key tool in approaching the relationship between a module and its endomorphism algebra is to utilise idempotents in the latter. Our first two results hold for modules over arbitrary rings and the straightforward proofs may be found in [6, Section 106 (c) (d)].

Proposition 2.1. If $M$ is a module over an arbitrary ring $S$ and $e$ is an idempotent in $E(M)$, then the rings eE(M)e and $E(e M)$ are isomorphic.

Proposition 2.2. Suppose $A, A^{\prime}$ are modules over an arbitrary ring $S$ and there is an algebra isomorphism $\Phi: E(A) \rightarrow E\left(A^{\prime}\right)$. If $A$ has a decomposition $A=B \oplus C$, then $A^{\prime}$ has a decomposition $A^{\prime}=B^{\prime} \oplus C^{\prime}$ and $\Phi$ induces isomorphisms $E(B) \cong E\left(B^{\prime}\right), E(C) \cong$ $E\left(C^{\prime}\right)$.

We will use these results repeatedly in our proofs and in the latter stages of the paper we will often refer to such usage as 'by the standard argument'.

The next result also holds for modules over an arbitrary ring $S$ and is based on an idea of Corner which we have previously exploited in [9].

Lemma 2.3. Suppose that $\Phi$ is an algebra isomorphism between the endomorphism algebras $E(G), E(H)$. Let $\alpha_{1}, \alpha_{2}$ be projections of $G$ onto the summands $A_{i}=\alpha_{i}(G),(i=$ $1,2)$ and let $\beta_{i}=\Phi\left(\alpha_{i}\right)$ be the corresponding projections of $H$ onto summands $B_{i}=$ $\beta_{i}(H),(i=1,2)$. Then if $\operatorname{Hom}\left(A_{1}, A_{2}\right)=0$ so too is $\operatorname{Hom}\left(B_{1}, B_{2}\right)$.

Proof. Let $\beta: B_{1} \rightarrow B_{2}$ be an arbitrary homomorphism and extend it to an endomorphism of $H$ by setting $\beta(1-\beta) 1)(H)=0$. Observe that if $x$ is an arbitrary element of $H$ then $\beta(x)=\beta\left(\beta_{1}(x)+\left(1-\beta_{1}\right)(x)=\beta \beta_{1}(x)\right.$ so that $\beta=\beta \beta_{1}$. Moreover, as $\beta(x) \in B_{2}, \beta_{2} \beta(x)=\beta(x)$ from which it follows that $\beta=\beta_{2} \beta \beta_{1}$. Taking images under the algebra-isomorphism $\Phi^{-1}$ we get that $\Phi^{-1}(\beta)=\alpha=\alpha_{2} \alpha \alpha_{1}$ and as this is a homomorphism : $A_{1} \rightarrow A_{2}$, we have that $\alpha=0$. But then $\beta=\Phi(\alpha)=0$, as required.

In our work in [9], we found it convenient and useful to introduce relationships that we termed the 'Fundamental Relations'; these relationships enabled us to transfer information from endomorphism algebras of decomposable modules into information relating to homomorphism modules. Suppose that $G, H$ are $R$-modules with $E(G) \cong$ $E(H)$ via an isomorphism $\Phi$ and $\pi$ is an idempotent of $E=E(G)$, so that from Proposition 2.2 we get an idempotent $\pi^{\prime} \in E(H)=E^{\prime}$ and $E \pi \cong E^{\prime} \pi^{\prime}$; similarly we also will have $\pi E \cong \pi^{\prime \prime} E^{\prime}$. However, the relationships $\operatorname{Hom}(\pi(G), G) \cong E \pi$ and 
$\operatorname{Hom}(G, \pi(G)) \cong \pi E$ always hold so we get the following relations for an idempotent $\pi \in E(G)$ and the corresponding image of $\pi$ under an algebra-isomorphism, $\pi^{\prime} \in E(H)$ :

$$
\operatorname{Hom}(\pi(G), G) \cong \operatorname{Hom}\left(\pi^{\prime}(H), H\right) \text { and } \operatorname{Hom}(G, \pi(G)) \cong \operatorname{Hom}\left(H, \pi^{\prime}(H) .\right.
$$

We remark that in the situation where $E(G)$ and $E(H)$ are anti-isomorphic as in [9], these relationships are 'twisted' and result in quite strong restrictions on $G, H$. Surprisingly, in the current situation we shall only need to appeal to this result in the proof of Proposition 3.1 ; otherwise Lemma 2.3 will suffice for our purposes.

As a first application of the ideas above we wish to consider the relationship between modules and their endomorphism algebras in some simple cases, including the case of a vector space and its ring of linear transformations. To derive a uniform approach which we believe illustrates the fundamental idea, we introduce an ad hoc concept: a cyclic direct summand, generated by $x$ say, of an $R$-module $G$ is said to be an endo-root if, given any $y \in G$, there is an endomorphism of $G, \phi_{y}$ say, with $\phi_{y}(x)=y$.

Note that in a vector space over a field, every non-zero element is an endo-root and for a reduced $R$-module $G$, any summand isomorphic to $R$ has a generator which is an endo-root. It is also reasonably straightforward to show that if $G$ is is a bounded $R$-module then any element of exponent equal to that of $G$ is also an endo-root: such an element generates a direct summand - see, for example [11, Lemma 4] - and the maximality of order of such an element permits one to define an endomorphism of $G$ mapping this element onto any other element of $G$. A simple example of an $R$-module without an endo-root is the direct sum of the cyclic modules $R\left(p^{n}\right)$, one copy for each positive integer $n$.

Theorem 2.4. Let $G, H$ be reduced $R$-modules and suppose that $G$ has an endo-root $x$ with $R x=\pi(G)$ for some idempotent $\pi \in E(G)$. If $\Phi: E(G) \rightarrow E(H)$ is an algebra isomorphism with the property that $\Phi(\pi)(H)$ is generated by an endo-root of $H$, then $G \cong H$.

Proof. If $G$ is indecomposable then either (i) $G=R$ or (ii) $G=R\left(p^{n}\right)$ for some positive integer $n$. In either case we have that $E(G) \cong G$ and $E(G)$ is an integral domain. Thus in either case as $E(H) \cong E(G)$, we must have that $H$ is indecomposable as its endomorphism ring is an integral domain. In case (i) $E(H) \cong R$ and, as observed in the introduction, $H$ is either $R$ or $Q / R$, with the latter being excluded since, by hypothesis, $H$ is reduced so that $H \cong G$. In case (ii) the only possibility for $H$ is $R\left(p^{n}\right)$ and again $H \cong G$.

Now if $G$ is decomposable we have $G=A \oplus B$ for $A=\langle x\rangle$ and some $B \neq 0$. Then it follows from Proposition 2.2 that there is a decomposition $H=A^{\prime} \oplus B^{\prime}$ with $E(A) \cong$ $E\left(A^{\prime}\right), E(B) \cong E\left(B^{\prime}\right)$; furthermore, we have that $A^{\prime}=\Phi(\pi)(H)$ so by hypothesis $A^{\prime}=\langle z\rangle$ for some endo-root $z$ of $H$.

Given any $g \in G$, we can find an endomorphism $\alpha$ of $G$ with $\alpha(x)=g$. Now define a map $\phi: G \rightarrow H$ by setting

$$
\phi(g)=(\Phi(\alpha))(z) .
$$

The first thing we must check is that $\phi$ is well defined, so suppose that there is another endomorphism $\beta$ with $g=\beta(x)$. Then $(\alpha-\beta)(x)=0$, so that $(\alpha-\beta) \pi$ is the zero map in $E(G)$ and hence $\Phi((\alpha-\beta) \pi)=\Phi(\alpha-\beta) \Phi(\pi)$ is the zero map in $E(H)$. Thus $\Phi(\alpha-\beta)(z)=0$ so that $\Phi(\alpha)(z)=\Phi(\beta)(z)$ and the mapping $\phi$ is well defined.

Since $\Phi$ is additive, so too is $\phi$; it remains to show that $\phi$ is bijective.

Suppose then that $\phi(g)=0$ for some $g \in G$, so that $\Phi(\alpha)(z)=0$. Since $\Phi(\pi)(x)=z$ this means that $\Phi(\alpha) \Phi(\pi)=0$ and, as $\Phi$ is an algebra isomorphism, $\Phi(\alpha \pi)=0$, from which it follows that $\alpha \pi$ is the zero map in $G$. Hence $g=\alpha(x)=\alpha \pi(x)=0$ and $\phi$ is monic. 
Finally let $h \in H$ be arbitrary. Since $z$ is, by hypothesis, an endo-root of $H$, there is an endomorphism, $\eta^{\prime}$ say, with $\eta^{\prime}(z)=h$. Let $\eta=\Phi^{-1}\left(\eta^{\prime}\right) \in E(G)$ and set $g=\eta(x)$. Observe that $\phi(g)=\Phi(\eta)(z)=\eta^{\prime}(z)=h$, so that $\phi$ is surjective .

We remark that the restriction to $R$-modules is not necessary and a key idea for a more general result is that we want our modules to have the property that two indecomposable modules are isomorphic if and only if their endomorphism algebras are isomorphic.

Corollary 2.5. If $V, W$ are vector spaces over a field and the rings of linear transformations are isomorphic as rings, then $V \cong W$.

Proof. If $V$ is a vector space then the argument in the first paragraph of the proof of Theorem 2.4 simplifies to the single possibility that $G$ is isomorphic to the field itself. Furthermore, as observed above, every non-zero element of a vector space is an endoroot, so the requirement in Theorem 2.4 that $\Phi(\pi)$ gives an endo-root in $W$ is trivially satisfied. The result then follows directly from Theorem 2.4.

Corollary 2.6. Let $G, H$ be reduced $R$-modules such that $G$ has a direct summand isomorphic to $R$. If $E(G) \cong E(H)$, then $G \cong H$. In particular, if $G, H$ are reduced torsion-free $R$-modules with isomorphic endomorphism algebras, then $G, H$ are isomorphic.

Proof. This follows immediately from Theorem 2.4 and our earlier observation that a generator of a summand isomorphic to $R$ is always an endo-root.

Corollary 2.7. If $G, H$ are bounded $R$-modules with $E(G) \cong E(H)$, then $G \cong H$.

Proof. Observe firstly that if $G$ is $p^{n}$-bounded and $\Phi: E(G) \rightarrow E(H)$ is an algebra isomorphism, then $H$ is also $p^{n}$-bounded: if $p^{n} G=0$ then $p^{n} E(G)=0$ and the isomorphism $\Phi$ then forces $p^{n} E(H)=0$, giving $p^{n} 1_{H}=0$ and hence $p^{n} H=0$. Now choose an element $x \in G$ of maximal order and note, as observed above, that such an element $x$ is an endo-root of $G$. If $\pi: G \rightarrow\langle x\rangle$ is a projection, then $\Phi(\pi)$ gives rise to a summand $\langle z\rangle$ of $H$ with $E(\langle x\rangle) \cong E(\langle z\rangle)$, so that $\langle z\rangle$ is also an element of order $p^{n}$, and hence a summand of $H$. The maximality of its order then means that $\langle z\rangle$ is an endo-root of $H$ and the result now follows directly from Theorem 2.4.

\section{Modules Which ARE NOT REDUCED}

In this section we consider $R$-modules which are not reduced and show that we can restrict our considerations to reduced modules. We begin by investigating the endomorphism algebras of divisible $R$-modules.

Proposition 3.1. Let $G$ be a divisible $R$-module and $H$ an $R$-module with $E(G) \cong$ $E(H)$. Then

(i) If $G=\bigoplus_{\lambda} Q$ for some non-zero cardinal $\lambda$, then $H \cong G$;

(ii) If $G=\bigoplus_{\mu} Q / R$ for some non-zero cardinal $\mu$, then either $H \cong G$ or $H=\widehat{\bigoplus_{\mu} R}$;

(iii) If $G=\bigoplus_{\lambda} Q \oplus \bigoplus_{\mu} Q / R$ with $\lambda, \mu$ non-zero cardinals, then $G \cong H$.

Proof. (i) If $G$ is torsion-free divisible then it follows from standard properties of homomorphism groups that $E(G)$ is torsion-free and divisible as an $R$-module. But then $H$ cannot have a summand isomorphic to any of $R, Q / R$ or $R\left(p^{n}\right)$ since an application of Proposition 2.2 would result in the contradiction that $E(G)$ has such a summand. 
(ii) Let $\pi$ be a projection of $G$ onto a single summand isomorphic to $Q / R$ and denote by $\pi^{\prime}$ the corresponding projection in $E(H)$. Then since $E(\pi(G)) \cong R \cong E\left(\pi^{\prime}(H)\right)$, we have that $\pi^{\prime}(H)$ is either $R$ or $Q / R$. By the first of our fundamental relations we get that $\operatorname{Hom}\left(Q / R, \bigoplus_{\mu} Q / R\right)$ is isomorphic to either $\operatorname{Hom}(R, H)$ or $\operatorname{Hom}(Q / R, H)$. We know from [5, Proposition 44.3] that $\operatorname{Hom}\left(Q / R, \bigoplus_{\mu} Q / R\right) \cong \widehat{\bigoplus_{\mu} R}$ so that $H \cong$ $\operatorname{Hom}(R, H) \cong \widehat{\bigoplus_{\mu} R}$.

Suppose then $\pi^{\prime}(H) \cong Q / R$. Note firstly that $H$ cannot have a summand isomorphic to $Q$ since this would result in the contradiction that $G$ has such a summand. So if $H=D \oplus X$ with $D$ divisible and $X$ reduced, then $D \cong \bigoplus_{\alpha} Q / R$ for some cardinal $\alpha$. Furthermore, $X$ is reduced and $\operatorname{so} \operatorname{Hom}(Q / R, H)=\operatorname{Hom}(Q / R, D) \cong \widehat{\bigoplus_{\alpha} R} \cong \widehat{\bigoplus_{\mu} R}$. Reducing modulo $p$ and taking vector space dimensions we get that $\alpha=\mu$. It remains to show that $X$ is necessarily zero. If not, then we have a decomposition of $H=D \oplus X$ and $\operatorname{Hom}(D, X)=0$ and this would result in a decomposition of $G$ of the form $G=A \oplus B$ with $A, B$ both non-zero and, by $\operatorname{Lemma} 2.3, \operatorname{Hom}(A, B)$ would have to be zero - this is impossible since any summand of $G$ is necessarily a sum of copies of $Q / R$.

(iii) Let $\pi$ be a projection from $G$ onto $\bigoplus_{\mu} Q / R$ along $Y=\bigoplus_{\lambda} Q$. Then from Proposition 2.2 we get a projection $\pi^{\prime}$ of $H$ and a decomposition $H=\pi^{\prime}(H) \oplus X$, and $E(X) \cong E(Y), E(\pi(G)) \cong E\left(\pi^{\prime}(H)\right)$; note that it follows from part(i) that $X \cong Y$. It follows from part (ii) that $\pi^{\prime}(H)$ is isomorphic to either $\bigoplus_{\mu} Q / R$ or to $\widehat{\bigoplus_{\mu} R}$. However, $\operatorname{Hom}(\pi(G), Y)=0$ while if the second possibility holds, $\operatorname{Hom}\left(\pi^{\prime}(H), X\right)$ contains a copy of $\operatorname{Hom}(R, X)$ and is non-zero, contrary to Lemma 2.3. So the only possibility is that $\pi^{\prime}(H) \cong \bigoplus_{\mu} Q / R$ and $H \cong G$.

With the information from Proposition 3.1 at hand we can now take the necessary steps to reduce our problem to one concerning reduced modules only. First we need a simple fact regarding the four types of indecomposable $R$-module.

Lemma 3.2. If $X$ is an indecomposable $R$-module then $\operatorname{Hom}(X, Q / R) \neq 0$.

Proof. Since $Q / R$ contains a copy of $R\left(p^{n}\right)$ for every positive integer $n$, the result is immediate if $X=R\left(p^{n}\right)$; if $X=R$ then $\operatorname{Hom}(X, Q / R)$ is isomorphic to $Q / R$ itself, while if $X=Q / R$ then the homomorphism module is $R$. The final case to consider is then $X=Q$. Apply the functor $\operatorname{Hom}(Q,-)$ to the short exact sequence $0 \rightarrow R \rightarrow Q \rightarrow$ $Q / R \rightarrow 0$ and note that the completeness of $R$ forces the term $\operatorname{Ext}^{1}(Q, R)$ to be zero, so that $\operatorname{Hom}(Q, Q) \cong \operatorname{Hom}(Q, Q / R) \neq 0$.

So suppose now that $G$ is an $R$-module of the form $G=D \oplus G_{1}$ where $D$ is divisible, $G_{1}$ is reduced and both are non-zero. If $H$ is an $R$-module with $E(G) \cong E(H)$ then:

(a) If $D=\bigoplus_{\lambda} Q$ and $\pi$ is the projection of $G$ onto $D$ along $G_{1}$, then it follows from Proposition 2.2 that $H$ decomposes as $H=D^{\prime} \oplus H_{1}$ with $E(D) \cong D^{\prime}$ and $E\left(G_{1}\right) \cong$ $E\left(H_{1}\right)$; it now follows from Proposition 3.1 that $D \cong D^{\prime}$. Furthermore, if $H_{1}$ has a summand isomorphic to $Q$, then a further application of Proposition 2.2 to $H_{1}$ would give that $G_{1}$ has a summand isomorphic to $\mathrm{Q}$, contrary to $G_{1}$ being reduced. On the other hand, if $H_{1}$ has a summand isomorphic to $Q / R$ then $\operatorname{Hom}\left(\bigoplus Q, H_{1}\right) \geq$ $\operatorname{Hom}\left(\bigoplus_{\lambda} Q, Q / R\right) \neq 0$, the last inequality coming from Lemma 3.2. Now $G \stackrel{\lambda}{=} \bigoplus_{\lambda} Q \oplus G_{1}$ 
and $\operatorname{Hom}\left(\bigoplus_{\lambda} Q, G_{1}\right)=0$ since $G_{1}$ is reduced. An application of Lemma 2.3 would then force $\operatorname{Hom}\left(\bigoplus_{\lambda} Q, H_{1}\right)=0$, contrary to Lemma 3.2. So we conclude that $H_{1}$ does not have a summand isomorphic to $Q / R$ and it then follows that $H=D^{\prime} \oplus H_{1}$ with $D^{\prime} \cong D$ and $H_{1}$ is reduced with $E\left(H_{1}\right) \cong E\left(G_{1}\right)$.

(b) If $D=\bigoplus_{\mu} Q / R$ then the argument used in part (a) gives us that $H=D^{\prime} \oplus H_{1}$ with $E(D) \cong E\left(D^{\prime}\right)$ and $E\left(H_{1}\right) \cong E\left(G_{1}\right)$; note that as $G_{1}$ is non-zero, so too is $H_{1}$. In this case an application of Proposition 3.1 yields two possibilities: either $D^{\prime} \cong D$ or $D^{\prime} \cong \widehat{\bigoplus_{\mu} R}$. Now $\operatorname{Hom}\left(D, G_{1}\right)=0$ so that by Lemma 2.3 we must have $\operatorname{Hom}\left(D^{\prime}, H_{1}\right)=$ 0. This, however, is impossible if $D^{\prime} \cong \widehat{\bigoplus_{\mu} R}$, since the last term has a summand isomorphic to $R$. Thus we must have that $G=\bigoplus_{\mu} Q / R \oplus G_{1}, H=\bigoplus_{\mu} Q / R \oplus H_{1}$ and $E\left(G_{1}\right) \cong E\left(H_{1}\right)$. We claim that in this case $H_{1}$ is also reduced.

Clearly the standard argument shows that $H_{1}$ cannot have a summand isomorphic to $Q$ since $G_{1}$ is reduced. Furthermore, as $\operatorname{Hom}\left(\bigoplus_{\mu} Q / R, G_{1}\right)=0$ it follows from Lemma 2.3 that $\operatorname{Hom}\left(\bigoplus_{\mu} Q / R, H_{1}\right)=0$ and this is impossible if $H_{1}$ contains a summand isomorphic to $Q / R$. So $H_{1}$ is reduced as claimed, and $G=\bigoplus_{\mu} Q / R \oplus G_{1}, H=\bigoplus_{\mu} Q / R \oplus$ $H_{1}$ and $E\left(G_{1}\right) \cong E\left(H_{1}\right)$.

The final reduction we need relates to the following situation:

(c) If $D=\bigoplus_{\lambda} Q \oplus \bigoplus_{\mu} Q / R$ with both $\lambda, \mu$ non-zero, then applying the standard argument to the projection of $G$ onto $D$ along $G_{1}$, we get that $H$ decomposes as $H=$ $D^{\prime} \oplus H_{1}$ with $E(D) \cong E\left(D^{\prime}\right)$ and $E\left(G_{1}\right) \cong E\left(H_{1}\right)$. It follows from Proposition 3.1 (iii) that $D^{\prime} \cong D$. An identical argument to that in part (b) shows that $H_{1}$ is reduced.

In summary then we have established the following:

Theorem 3.3. Let $G=D \oplus G_{1}$ with $D$ non-zero divisible and $G_{1}$ non-zero reduced and suppose $H$ is an $R$-module with $E(G) \cong E(H)$, then $H=D^{\prime} \oplus H_{1}$ with $D^{\prime} \cong D$ and $H_{1}$ is reduced with $E\left(H_{1}\right) \cong E\left(G_{1}\right)$.

So we may restrict our considerations to reduced modules in the sequel.

\section{Reduced modules}

Suppose now that $G, H$ are reduced $R$-modules with $E(G) \cong E(H)$. We examine the various possibilities; firstly we consider the situation in which $G$ has a direct summand isomorphic to $R$. Since $H$ is also reduced we see from our previous arguments that $H$ must also have such a summand and now it follows from Corollary 2.6 that $G$ and $H$ are isomorphic.

Now consider the situation where $G$, and hence of course $H$, does not have a summand isomorphic to $R$. This means that a basic submodule of $G$ is torsion and similarly for $H$. However, the fact that a basic submodule is torsion does not necessarily mean that the module itself is torsion. In the situation where $E(G) \cong E(H)$, we can derive some additional information: a torsion basic submodule is of the form $B=\bigoplus_{n=1}^{\infty} B_{n}$ where each $B_{n}$ is a direct sum of cyclic $R$-modules $R\left(p^{n}\right)$ and the $B_{n}$ are summands of the module. Consequently our standard argument using projections onto these summands in $G$ will yield corresponding summands in $H$ which are isomorphic and it is then easy to see that $G, H$ may be regarded as having a common basic submodule. In fact we can 
say a good deal more as evidenced by our next result, a proof of which can be found in [9, Proposition 3.2].

Proposition 4.1. If $G$ is a reduced $R$-module which does not have a direct summand isomorphic to $R$ and $B$ is a basic submodule of $G$, then (i) $G / t G$ is divisible;

(ii) $G / B \cong(t G / B) \oplus D$ where $t G / B$ is torsion divisible and $D$ is torsion-free divisible and isomorphic to $G / t G$.

There are two possibilities to consider: $G$ is torsion or $G$ is mixed. This in turn leads to two cases which we will consider separately: (a) both $G$ and $H$ are torsion or (b) at least one of $G, H$ is mixed.

In case (a) we can appeal to the celebrated theorem of Kaplansky - see [11, Theorem 28] or [7, Theorem 16.2.5] - mentioned in the introduction which tells us that $G \cong H$.

In case (b) we have just seen that $G, H$ are extensions of a common $R$-module which is a direct sum of cyclic modules by a divisible $R$-module. Despite this, the possibility of obtaining a complete answer to our original question is totally dashed by the next example. This example has already appeared in [2, Theorem 3.2] and we paraphrase below the relevant part of that result.

Theorem 4.2. There exists a mixed $R$-module $G$ and a family of submodules $G(\psi)$ indexed by a real parameter $\psi, 0<\psi \leq 1$ such that

(a) $G(\psi) \varsubsetneqq G\left(\psi^{\prime}\right)$ if $0<\psi<\psi^{\prime} \leq 1$;

(b) $E(G(\psi)) \cong E(G)$ for $0<\psi \leq 1$;

(c) $G(1)$ is the torsion-completion of a basic submodule of $G$.

Summarising all of the above we have established:

Theorem 4.3. If $G, H$ are $R$-modules with $E(G) \cong E(H)$ then $G \cong H$ unless

(i) $G=\bigoplus_{\mu} Q / R$ and $H=\widehat{\bigoplus_{\mu} R}$ for some cardinal $\mu$ or

(ii) $G, H$ have isomorphic torsion basic submodules $B$ and at least one of $G, H$ is a non-torsion extension of $B$ by a divisible $R$-module.

Remark 4.4. : The conditions on $G, H$ in Theorem 4.3 above are necessary conditions but condition(i) is also sufficient in the sense that if $G=\bigoplus_{\mu} Q / R$ and $H=\widehat{\bigoplus} \widehat{\bigoplus}_{\mu}$, then $E(G) \cong E(H)$ - see, for example the discussion of the Matlis Category Equivalence in [8, Section VIII.2] or [13].

\section{REFERENCES}

[1] R. Baer, Automorphism rings of primary abelian operator groups, Ann. Math., 44, (1943), 192-227.

[2] A. L. S. Corner, B. Goldsmith and S. L. Wallutis, Anti-isomorphisms and the failure of duality, in Models, Modules and Abelian groups (editors R. Göbel and B. Goldsmith) de Gruyter, Berlin-New York, 2008, 315-323.

[3] T.G. Faticoni, A new proof of the Baer-Kaplansky Theorem, Comms. Algebra, 19, (1991), 31193123.

[4] S.T. Files and W. J. Wickless, The Baer-Kaplansky theorem for a class of global mixed groups, Rocky Mt. J., 26, (1996), 593-613.

[5] L. Fuchs, Infinite Abelian Groups, Vol. I, Acad. Press, New York and London, 1970.

[6] L. Fuchs, Infinite Abelian Groups, Vol. II, Acad. Press, New York and London, 1973.

[7] L. Fuchs, Abelian Groups, Springer, Switzerland 2015.

[8] L.Fuchs and L. Salce, Modules over Non-Noetherian Domains, Mathematical Surveys and Monographs, Vol.84, American Math. Soc. 2001.

[9] B. Goldsmith and N.White, Corner's Theorem on Modules with Anti-isomorphic Endomorphism Algebras to appear in Periodica Math. Hungar. 
[10] G.J. Hauptfleisch, Torsion-free abelian groups with isomorphic endomorphism rings, Arch. Math. (Basel), 24, (1973), 269-273.

[11] I. Kaplansky, Infinite Abelian Groups, Univ. of Michigan Press, Ann Arbor, Michigan, 1954 and 1969.

[12] P.A. Krylov, A.A. Tuganbaev and A.V. Tsarev, Around the Baer-Kaplansky Theorem, J. Math. Sciences, 256, (2021), 278-298.

[13] W. Liebert, Characterization of the endomorphism rings of divisible torsion modules and reduced complete torsion-free modules over complete discrete valuation rings, Pacific J. Math., 37, (1971), 141-170.

[14] W. May and E. Toubassi, Endomorphisms of Abelian groups and the theorem of Baer and Kaplansky , J. Algebra, 43, (1976), 1-13.

[15] W. May and E. Toubassi, Classifying endomorphism rings of rank one mixed groups in Abelian Groups and Modules, CISM Courses and Lectures, Vol.287, Springer Berlin, 1984, 253-263.

[16] K.G. Wolfson, Isomorphism of the endomorphism rings of torsion-free modules, Proc. Amer. Math. Soc., 13, (1962), 712-714.

Brendan Goldsmith is former president of the IMS and DIT, currently professor emeritus at TU Dublin with research interests in algebra.

Noel White Was a research student working toward a $\mathrm{PhD}$ in mathematics. He passed away at the beginning of September, shortly after the submission of this paper. RIP.

(Both authors) School of Mathematical Sciences, Technological University Dublin

E-mail address: brendan.goldsmith@TUDublin.ie, noelwhite8@gmail.com 